Abstract IDDF2019-ABS-0087 Table 2 Results of data from two time periods

\begin{tabular}{|l|l|l|l|} 
& $\begin{array}{l}\text { Time 1 } \\
(2003-2010)\end{array}$ & $\begin{array}{l}\text { Time 2 } \\
(2011-2018)\end{array}$ & P-value \\
\hline Age & $\mathbf{N}=378$ & $\mathrm{~N}=296$ & NA \\
\hline Sex (Male) & $63 \pm 12.5$ & $66 \pm 12.5$ & 1.00 \\
\hline Sex (Female) & 250 & 255 & $>0.99$ \\
\hline HBV & 128 & 41 & $<0.05^{*}$ \\
\hline HCV & 247 & 104 & $<0.002^{*}$ \\
\hline NAFLD & 7 & 10 & $<0.2241$ \\
\hline ALD & 15 & 99 & $<0.0000001^{*}$ \\
\hline Unknown & 58 & 33 & $<0.035^{*}$ \\
\hline DIH & 0 & 45 & NA \\
\hline AIH & 5 & 0 & NA \\
\hline & 0 & 0 & NA \\
\hline
\end{tabular}

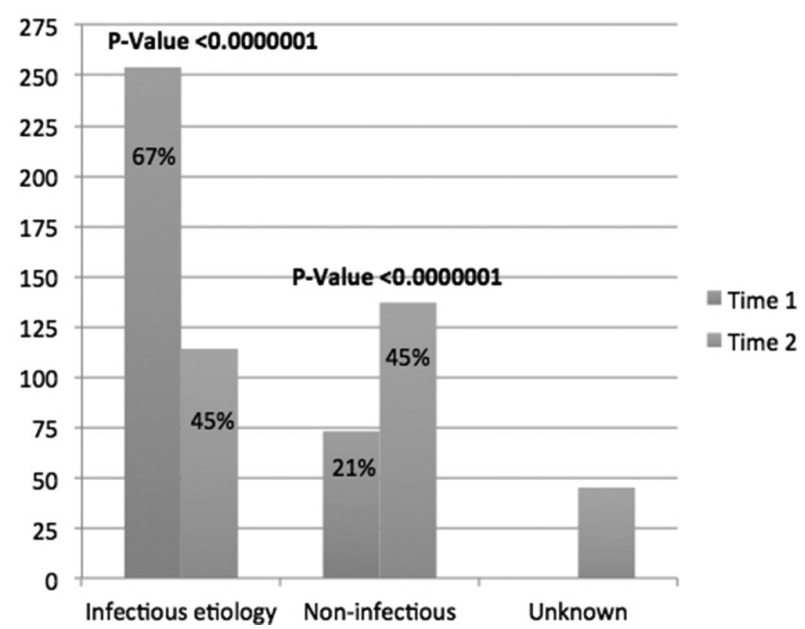

Abstract IDDF2019-ABS-0087 Figure 1 Comparison of etiology time 1 versus time 2

Conclusions - Hepatitis B is still the most common etiology of Hepatocellular Carcinoma

- There has been a significant increase in the development of HCC from non-infectious etiologies

- Of the non-infectious etiologies, NAFLD seems to be an emerging threat in the development of Liver Cancer

\section{IDDF2019-ABS-0092 HEPATOCELLULAR CARCINOMA WITH A RARE SCALP AND SKULL METASTASIS - A CASE REPORT}

Prima Bianca Chua*, Diana Payawal. Cardinal Santos Medical Center, Philippines

\subsection{6/gutjnl-2019-IDDFabstracts.260}

Background Hepatocellular Carcinoma (HCC) is the second leading cause of cancer death worldwide, especially in Asia and Africa.2-4 HCC commonly metastasizes to regional lymph nodes and lungs, and less commonly to bone.6 Cutaneous metastases are extremely rare with only a few reports in the literature.

Methods

- A 56-year-old male diagnosed case of chronic hepatitis B for 9 years previously on Adefovir.

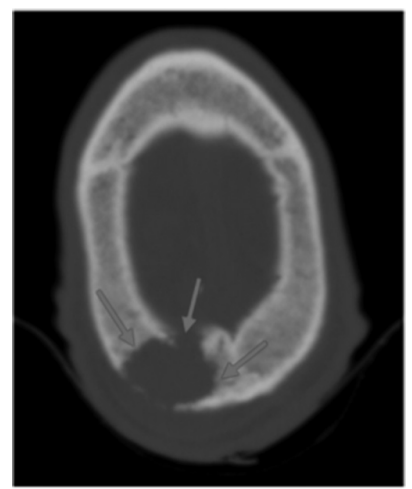

Abstract IDDF2019-ABS-0092 Figure 1 Bone window Lytic lesion was noted in the right parietal bone (arrows)

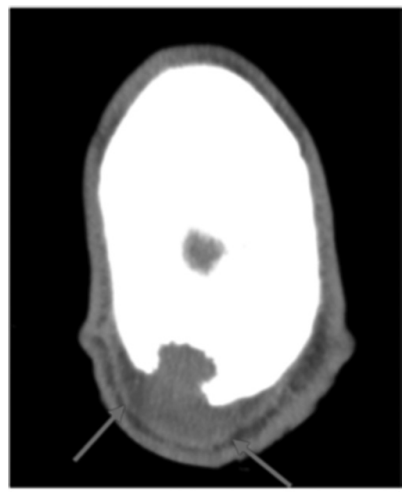

Abstract IDDF2019-ABS-0092 Figure 2 Soft tissue window. A soft tissue component of the lytic lesion was noted (arrows)

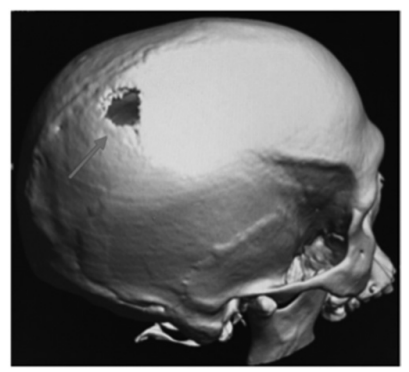

Abstract IDDF2019-ABS-0092 Figure 3 Three Dimensional (3D) window. A hollytic lesion was noted in the right parietal area (arrow)

- A magnetic resonance imaging of the liver with contrast revealed a mass in segment 5 and $6(11.2 \times 14.4 \times 10.8 \mathrm{~cm})$, and an elevated alpha fetoprotein $>1000 \mathrm{IU} / \mathrm{ml}$, he underwent transcatheter arterial chemoembolization.

- One week after the procedure he noted a painless parietal scalp lump and rapidly enlarging in a span of three weeks.

- Examination revealed a soft, fluctuant, subcutaneous lump in the right parietal area measuring $2.5 \times 3 \mathrm{~cm}$.

- A cranial CT scan with contrast revealed an enhancing expansile lytic lesion with a soft tissue component at the right parietal bone measuring $2.4 \times 3.5 \times 3.2 \mathrm{~cm}$ seen at both parietal bone shown in figures 1, 2 and 3 (figures 1,2, and 3 CT scan image of a patient with scalp and skull metastasis).

- He was given palliative management. He developed hepatorenal syndrome and eventually died on the fourth hospital day. 
Results Intrahepatic metastasis is the most common metastasis of HCC. Extrahepatic metastases usually occur in the regional lymph nodes (16\%-40\%) and lungs (34\%-70\%), but less commonly in the skeleton (1.6\%-16\%). In skeleton metastasis, common sites are a vertebral column, pelvis, femora, and ribs, but rarely the skull. The incidence of skull metastases from HCC is $0.4 \%-1.6 \%$. Among cases of skull metastases as the first symptom $(24 / 59,41 \%)$ and those with solitary skull metastases (14/ $59,24 \%)$ were identified. In all 24 cases, $71 \%$ were misdiagnosed because the incidence of skull metastasis is low. 15

Conclusions HCC is a debilitating condition leading to increased mortality and poor prognosis. It is important to educate patients with chronic hepatitis B to have regular follow-ups to catch early stages of HCC and provide appropriate treatment strategies, improve prognosis and quality of life. A high index of suspicion in patients with scalp lesion is warranted as this can be an initial presentation of HCC and decrease misdiagnosis rate.

\section{IDDF2019-ABS-0093 MICROWAVE ABLATION IN HEPATOCELLULAR CARCINOMA: CASE SERIES}

Prima Bianca Chua*, Diana Payawal, John Alfredo Raphael Pangilinan. Cardinal Santos Medical Center, Philippines

10.1136/gutjnl-2019-IDDFabstracts.261
Background Hepatocellular Carcinoma (HCC) is the second leading cause of cancer death worldwide. (1-2) Current treatment guidelines recommend surgical resection or liver transplantation as the gold standard treatment for very early and early-stage HCC patients with well-conserved liver function. (3-5) Majority of patients who are diagnosed with HCC are not eligible for said treatments thus thermal ablative therapies have emerged as a well-accepted alternative treatment option. (6-9) Among thermal ablative techniques, radiofrequency ablation (RFA) is currently the commonly used and has emerged as a curative treatment for early-stage HCC beyond resection and liver transplantation. (6,8) Microwave ablation (MWA), another thermal ablative technique, is similar to RFA but with a significant advantage. (17)

Methods We present 6 cases of HCC secondary to non-alcoholic fatty liver disease (NAFLD) and chronic hepatitis B with Child-Pugh A or B admitted in Cardinal Santos Medical Center. (figure 1).

Results In the Philippines, the majority of Hepatologists still use RFA in the management of early-stage HCC. The inability to truly quantify the extent of destruction especially in highly vascular tissue and is affected by 'heat sink' are few of its disadvantages.

MWA provides a more consistent and precise ablative technique through its mechanism of direct hyperthermic injury. The ability to precisely predict the ablation zones would be revolutionary in ensuring complete destruction of malignant tissue. The use of a MWA machine with predictable spherical

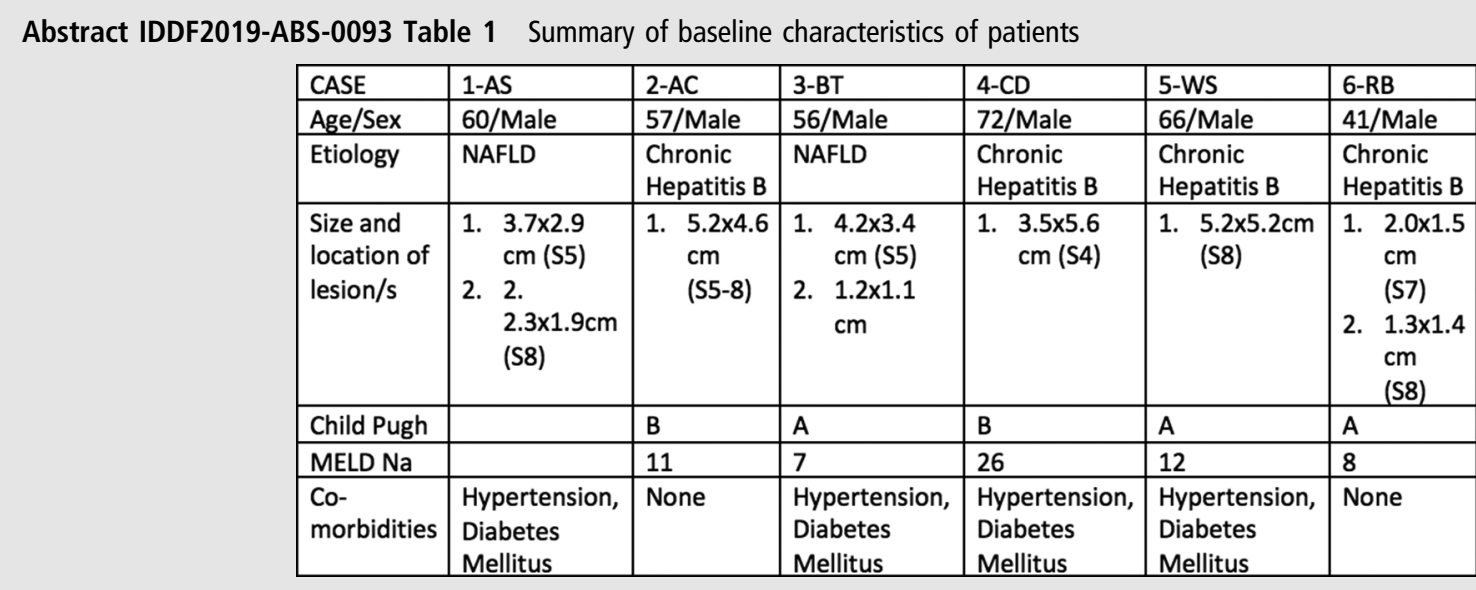
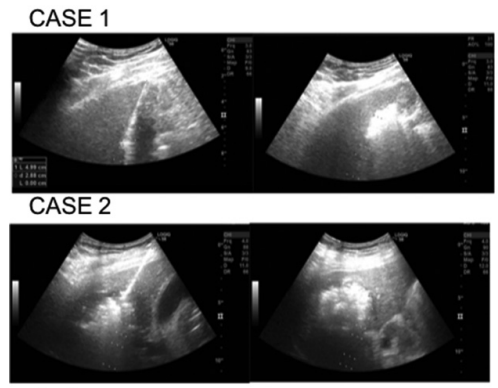

CASE 3

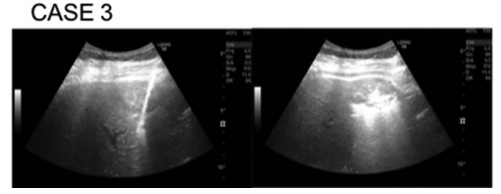

CASE 4

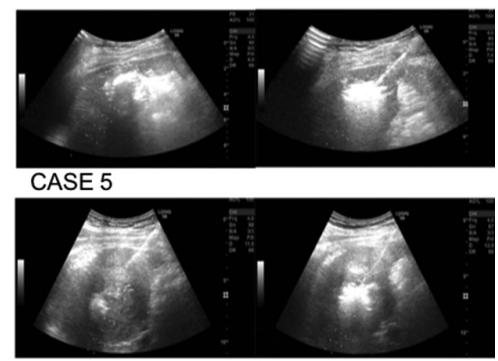

CASE 6

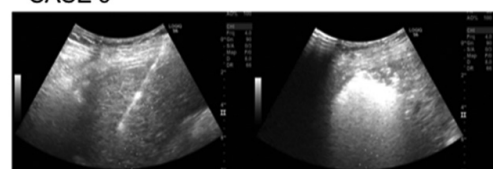

